Revue d'histoire de l'Amérique française

Q4. REVUE D'HISTOIRE DE L'AMÉRIQUE FRANÇAISE

\title{
Le régionalisme mauricien des années trente
}

\section{René Verrette}

Volume 47, numéro 1, été 1993

URI : https://id.erudit.org/iderudit/305181ar

DOI : https://doi.org/10.7202/305181ar

Aller au sommaire du numéro

\section{Éditeur(s)}

Institut d'histoire de l'Amérique française

ISSN

0035-2357 (imprimé)

1492-1383 (numérique)

Découvrir la revue

Citer cet article

Verrette, R. (1993). Le régionalisme mauricien des années trente. Revue d'histoire de l'Amérique française, 47(1), 27-52. https://doi.org/10.7202/305181ar

\section{Résumé de l'article}

Cet article présente le fruit d'une recherche sur les origines du régionalisme en Mauricie qui s'est exprimé avec vigueur durant les années trente. L'idéologie de quatre régionalistes ayant en tête Albert Tessier est mise en contexte avec l'évolution socio-économique de la Mauricie durement affectée par la crise de 1929. Nous cherchons à établir un lien entre les représentations du discours régionaliste et un besoin d'identification à un idéal mobilisateur engendré par une situation collective difficile. Nous cherchons de plus à mesurer jusqu'à quel point ce discours a constitué un élément moteur du développement régional à cette époque. Le discours est présenté en interaction avec les mouvements collectifs qui en découlent et l'ont nourri. Les influences françaises sont mises en relief de même qu'une résistance des élites face à la modernité et à l'américanité. 


\title{
LE RÉGIONALISME MAURICIEN DES ANNÉES TRENTE ${ }^{1}$
}

\author{
RENÉ VERRETTE \\ Centre interuniversitaire d'études québécoises \\ Université du Québec à Trois-Rivières
}

\section{RÉSUMÉ}

Cet article présente le fruit d'une recherche sur les origines du régionalisme en Mauricie qui s'est exprimé avec vigueur durant les années trente. L'idéologie de quatre régionalistes ayant en tête Albert Tessier est mise en contexte avec l'évolution socio-économique de la Mauricie durement affectée par la crise de 1929. Nous cherchons à établir un lien entre les représentations du discours régionaliste et un besoin d'identification à un idéal mobilisateur engendré par une situation collective difficile. Nous cherchons de plus à mesurer jusqu'à quel point ce discours a constitué un élément moteur du développement régional à cette époque. Le discours est présenté en interaction avec les mouvements collectifs qui en découlent et l'ont nourri. Les influences françaises sont mises en relief de même qu'une résistance des élites face à la modernité et à l'américanité.

\section{ABSTRACT}

This paper deals with the beginnings of a militant regionalism that expressed itself in Mauricie (Québec) during the thirties and analyzes the ideology of four regionalists with Albert Tessier at their head in relation to the social and economic evolution of a region hit hard by the Great Depression. We are looking for a link between the representations of a regional discourse motivating an ideal produced by a difficult situation. Moreover, we are trying to evaluate the degree to which this discourse has stimulated the regional development of the time. We present the discourse in constant interaction with the collective activities it helped to arise and develop. Finally, we stress the French influence over this regionalism as well as the distrust expressed by the local elite for modernity and americanism.

1. Le présent article reprend notre mémoire de maîtrise en études québécoises, Le régionalisme mauricien des années trente, Université du Québec à Trois-Rivières, ix-351 p. Que soient vivement remerciés les directeurs de la recherche, messieurs Guildo Rousseau et René Hardy du Centre d'études québécoises. La recherche s'est appuyée sur le dépouillement de journaux, de périodiques et de fonds d'archives du Centre de documentation en études québécoises de l'UQTR et du Séminaire Saint-Joseph de Trois-Rivières. Les répertoires de sources journalistiques et la bio-bibliographie d'Albert Tessier par Lucienne Leduc ont grandement aidé au repérage des éléments du corpus. Le témoignage de quelques contemporains a permis d'éclairer certains aspects obscurs des événements. Des remerciements s'adressent notamment à Clément Marchand.

RHAF, vol. 47, nº 1, été 1993 
La Mauricie, par la voix de ses élites, s'est préoccupée d'un problème d'identité qui a déterminé certaines composantes de l'histoire de son développement. Dans Forêt et société en Mauricie ${ }^{2}$, René Hardy et Normand Séguin notent que la Mauricie, avec Trois-Rivières comme chef-lieu régional, est née au XIX ${ }^{\mathrm{e}}$ siècle à la suite de l'exploitation commerciale de la forêt et de l'économie du bois. Néanmoins, font-ils remarquer, la zone trifluvienne apparaissait déjà, sous le Régime français, comme une entité distincte grâce à la division administrative d'un gouvernement spécifiant une ébauche d'identité régionale ${ }^{3}$. Par ailleurs, ajoutent les deux historiens, «la Mauricie n'est pas qu'un espace économique, elle existe aussi comme espace mental, comme une référence dans l'imaginaire collectif ${ }^{4} »$.

Effectivement, une prise de conscience régionale, développée depuis le siècle dernier, a connu une intensité particulière durant les années trente à partir de la réflexion de certaines élites locales recrutées dans le clergé, les professions libérales et le journalisme. Mise en branle par la petite bourgeoisie trifluvienne francophone, cette cristallisation a donné lieu à l'énonciation d'un discours que l'on désigne sous le nom de «régionalisme mauricien» - «régionalisme» parce que c'est le terme qu'employait son principal diffuseur, l'abbé Albert Tessier, et «mauricien» parce que ce discours s'adressait en premier lieu aux habitants de la Mauricie et qu'il moussait la fierté de vivre dans cette région. En 1938, Albert Tessier définissait ainsi le mouvement d'idée et d'action qu'il avait lancé dix ans auparavant: «Le régionalisme constitue la mise en valeur intelligente, ordonnée, méthodique des virtualités matérielles et spirituelles que le bon Dieu a mises à notre disposition dans un milieu à une époque donnée ${ }^{5}$.»

Le mouvement amorcé par Tessier peut être corrélé avec des événements sociaux et intellectuels qui ont constitué le fond de scène de la société mauricienne des années trente.

Le discours régionaliste mauricien s'est diffusé par la médiation d'associations culturelles, de débats intellectuels et de manifestations de la culture populaire. L'idée centrale est que la création du régionyme «Mauricie» et la polémique qui entoura son adoption, le déroulement des fêtes du Tricentenaire, la production culturelle élaborée autour de la mystique du flambeau ainsi que l'ensemble des créations culturelles issues de l'exploitation des thèmes régionalistes, toute cette

2. René Hardy et Normand Séguin, Forêt et société en Mauricie (Montréal et Ottawa, Boréal Express/Musée national de l'Homme, 1984), 5.

3. Ibidem, 8 .

4. Ibid., 204.

5. Le Nouvelliste, 26 novembre 1938. 
effervescence socioculturelle et idéologique de la Mauricie de 19301940 se présente comme le reflet, dans l'ordre du symbolique, d'une dynamique régionale fondée sur une spécificité solidement assise. Le présent article étudie l'émergence d'une idéologie régionaliste et sa diffusion en rapport avec le contexte social et économique provoqué par l'expansion accélérée de l'industrialisation mauricienne puis secoué par la crise des années trente. Nous cherchons à établir un lien entre l'inscription du régionalisme dans l'imaginaire collectif et un besoin d'évasion, de repli ou d'identification à un idéal mobilisateur engendré par une situation collective difficile. Cet article vise également à mesurer jusqu'à quel point l'idéologie en question a constitué un élément moteur du développement régional. Nous posons comme postulat de départ que le contexte économique agissant comme cause primordiale de l'évolution sociale est occulté par des représentations sociales cristallisées dans l'idéologie. Plus explicitement, la crise économique ressentie à partir de 1930 a constitué le catalyseur d'une tendance déjà amorcée lors de l'industrialisation rapide de la Mauricie depuis le tournant du $\mathrm{XX}^{\mathrm{e}}$ siècle.

Nous examinerons successivement le cadre économique et social de la Mauricie, le discours des principaux définisseurs du régionalisme ${ }^{6}$ et les actions collectives qui peuvent lui être rapportées avant d'élaborer la synthèse permettant d'éclairer la portée véritable de ce mouvement régional.

\section{I - LE CADRE ÉCONOMIQUE ET SOCIAL}

Le discours régionaliste se développe dans un contexte socioéconomique qu'il est impératif d'esquisser au moins à grands traits?

6. Le dépouillement a permis la constitution d'un corpus de référence constitué de 174 textes ou fragments de textes. Ceux d'Albert Tessier ont fait l'objet d'un traitement quantitatif à cause de leur ampleur. Pour les fins de l'analyse, n'ont été retenus que les extraits exposant l'idéologie régionaliste excluant les énoncés descriptifs. Les extraits retenus ont été rassemblés en cinq séries: 47 textes de Tessier sur le régionalisme mauricien, 47 textes d'autres auteurs sur le même sujet, 32 textes sur le régionalisme littéraire, 37 textes sur le régionyme Mauricie et 11 textes sur la mystique du flambeau. Chaque élément a été découpé en unité de signification correspondant à une phrase ou une partie de phrase complète en elle-même. Quatre cent un énoncés ont ainsi été relevés. Ce traitement a permis de mettre en relief une série de mots clés et de quantifier leur fréquence. Cette opération a donné lieu à la distinction de mots pivots autour desquels se sont groupés par affinité sémantique des mots annexes, lesquels sont le plus souvent des tournures périphrasiques plus ou moins synonymes. Ainsi, à «régionalisme» correspondent «fierté régionale», «réveil régionaliste», «amour de la petite patrie», «notre régionalisme» interchangeable dans les phrases où ils apparaissent.

7. Pour une esquisse de la socio-économie mauricienne durant l'époque à l'étude, voir René Hardy et Normand Séguin, op. cit.; Pierre Lanthier «Stratégie industrielle et développement régional», Revue d'histoire de l'Amérique française, 37,1 (juin 1983): 3-19; Pierre Lanthier et Alain Gamelin, L'industrialisation de la Mauricie. Dossier statistique et 
L'abondance de la matière première, la qualité des voies de communication et la proximité des marchés favorisent l'agglomération trifluvienne. Successivement, trois papetières, la Saint Maurice Paper (1911), la Wayagamack Pulp and Paper (1912) la Saint Lawrence Paper Mill (1923) s'implantent dans le Trois-Rivières métropolitain. De leur côté, la Wabasso Cotton commence sa production en 1908 et la Canada Iron Foundry agrandit ses installations afin d'être en mesure de fournir les chantiers de construction des papetières et des barrages électriques.

L'accélération de la croissance démographique constitue la conséquence la plus immédiate de l'expansion soudaine du secteur secondaire en Mauricie. Notamment, la population de la cité trifluvienne grimpe de 10000 en 1901 à 35000 trente ans plus tard. Par ailleurs, le transport et les voies de communication font l'objet d'une expansion similaire. Le réseau des chemins de fer est mis en place avant la Première Guerre et l'arrivée de l'automobile oblige les gouvernements à améliorer constamment le système routier. La route reliant Les Piles à La Tuque est ouverte à la circulation en 1925. Finalement, l'aménagement hydro-électrique du Saint-Maurice attire les industries consommatrices d'électricité qui recherchent les tarifs les plus bas.

Ainsi, la Mauricie connaît une période de développement qui la transforme presque complètement pendant les trois premières décennies du XX $\mathrm{XX}^{\mathrm{e}}$ siècle. Cependant le krach d'octobre 1929 a tôt fait d'exercer un impact négatif sur son expansion. L'agglomération trifluvienne subit rapidement, et avec une grande intensité, les contrecoups de la déstabilisation financière du monde occidental à cause de sa structure économique dominée par le secteur manufacturier.

Amorcé en 1930, l'effondrement des indicateurs économiques se poursuit à Trois-Rivières durant les années 1931-1932, pour atteindre un plancher en 1933. De 45 millions de dollars qu'elle est en 1929, la valeur de la production manufacturière brute chute à 16,5 millions, quatre ans plus tard. Si l'on compare ces données avec celles calculées pour l'ensemble de la Province, on constate que la décroissance est légèrement plus intense à Trois-Rivières. Les licenciements et les fermetures temporaires dans les grandes usines, les faillites de plusieurs entreprises de moindre envergure et le ralentissement général des affaires font surgir le spectre du chômage, avec son cortège de problèmes et d'humiliations. À l'automne 1930, la Ville de Trois-

chronologique 1870-1975 (Trois-Rivières, UQTR, Groupe de recherche sur la Mauricie, 1981) et Alain Gamelin, René Hardy, Jean Roy, Normand Séguin et Guy Toupin, Trois-Rivières illustrée (Trois-Rivières, Corporation des fêtes du $350^{\mathrm{e}}$ anniversaire de Trois-Rivières, 1984). 
Rivières instaure un système de "secours direct», contribuant à son financement pour un tiers, le reste étant partagé également entre Ottawa et Québec. Durant l'année 1933, environ le quart des Trifluviens ne peuvent compter que sur les maigres ressources du secours direct. Une lente remontée s'amorce alors et persiste jusqu'à la Seconde Guerre mondiale avec un léger repli en 1937.

L'exploitation forestière de l'arrière-pays, la stabilisation du front pionnier, consécutive à la saturation du terroir exploitable, l'équipement hydro-électrique du Saint-Maurice, la création de liens routiers et ferroviaires vers la Haute-Mauricie, l'arrivée massive de la grande industrie de type moderne et, finalement, l'accélération de l'urbanisation ont constitué autant de facteurs de transformations ayant déterminé une quête d'identité spécifique à la région. De fait, toutes ces transformations, autant sociales qu'économiques, rendaient plus impérieuse la nécessité d'intégrer les nouvelles représentations sociales imposées par l'accélération de l'histoire au sein d'une collectivité jusque-là habituée à une vision statique des choses. C'est dans ce contexte où s'accéléraient les changements sociaux que le discours régionaliste a pris corps et s'est imposé à la collectivité mauricienne.

\section{2 - LE DISCOURS RÉGIONALISTE}

Quatre acteurs individuels ont exercé une influence primordiale en élaborant et en diffusant le discours régionaliste mauricien. Deux d'entre eux sont membres du clergé, le troisième est médecin et le dernier, avocat. L'abbé Joseph-Gérin Gélinas ${ }^{8}$ joue un rôle d'animateur dans son enseignement de l'histoire au Séminaire Saint-Joseph à Trois-Rivières; il en va de même de l'abbé Albert Tessier ${ }^{9}$ qui succède à Gélinas durant les années vingt. Tessier est de loin celui qui a exercé l'activité régionaliste la plus forte parmi les quatre: à sa fonction

8. Neveu d'Antoine Gérin-Lajoie, Joseph-Gérin Gélinas naît à Louiseville en 1874. Ordonné en 1899, l'abbé Gélinas enseigne au Séminaire de Trois-Rivières de 1903 jusqu'à son décès survenu en 1927. Idéaliste et austère, il développa un amour de la petite patrie qu'il sut inculquer à ses élèves durant ses cours d'histoire. L'abbé Gélinas fut également un auteur prolifique qui signa des chroniques d'histoire dans les journaux et en rassembla un certain nombre dans deux recueils longtemps populaires dans les écoles: Au Foyer, causeries historiques pour les petits de chez nous et En veillant avec les petits de chez nous, 1: Régime français et 2: Régime anglais.

9. Né à Sainte-Anne-de-la-Pérade en 1895, Albert Tessier est ordonné en 1920. Il poursuit des études doctorales à Rome de 1920 à 1923 , complète sa formation à Paris en 1923 1924 et effectue un pèlerinage au pays de Frédéric Mistral en 1924. De retour à Trois-Rivières, il succède à Gélinas comme professeur en 1927. Il épouse la cause de l'histoire régionale jusqu'en 1937 (cf. supra) alors qu'il est nommé inspecteur des écoles ménagères et chargé d'un cours d'histoire à l'Université Laval. Il meurt à Trois-Rivières en 1976, laissant le souvenir d'un homme dynamique et très habile à défendre les idées qui ont guidé son action et sa carrière. 
d'enseignant, il a ajouté celle d'animateur social avant la lettre par des séries de conférences, des articles, des ouvrages, des films et des albums de photographies. Le $\mathrm{D}^{\mathrm{r}}$ Nérée Beauchemin ${ }^{10}$ s'est fait connaître comme poète, chantre ému et discret de «la petite patrie». Quant à $\mathbf{M}^{\mathrm{e}}$ Louis-Delavoie Durand ${ }^{11}$, candidat malchanceux à plusieurs élections, il a fait vibrer des auditoires par ses propos régionalistes d'ordre économique et politique.

Le terme «régionaliste» n'apparaît nulle part dans les écrits et les discours de l'abbé Gélinas, mais la ferveur avec laquelle il a vanté les vertus de la petite patrie à partir de 1910 dans Le Bien public le situe sans conteste comme précurseur immédiat d'Albert Tessier. Le témoignage de ses confrères de classe et de ses anciens élèves est unanime. Par exemple, Hector Héroux écrit: «Tout l'intéressait, tout le passionnait du moment qu'on parlait du Canada, de la petite patrie. Il consacrait des leçons entières à l'histoire de Trois-Rivières. [...] la petite histoire enchantait et retenait l'abbé Gélinas ${ }^{12} . »$ Pour Gélinas, le lien entre petite patrie et patriotisme national est direct, ce dernier constituant «la somme des patriotismes régionaux ${ }^{13}$ ». Toutefois, chez ce prêtre qui était un admirateur fervent de $\mathrm{M}^{\mathrm{gr}}$ Laflèche, la primauté du spirituel sur le temporel va de soi: l'amour de Dieu et de l'Église ont préséance sur celui de la patrie. Homme austère épris d'idéal, l'abbé Gélinas sème chez les élèves le goût du travail, de l'abnégation et du devoir. L'Église et la patrie sont partout présentes dans son discours régionaliste ${ }^{14}$ qu'il proclame avec ferveur lorsqu'il accompagne sa classe dans le Vieux Trois-Rivières. Également perçu comme un missionnaire de la pensée française, il s'emploie à la défense de «la gloire de la France» dans son interprétation des faits historiques ${ }^{15}$. Délaissant les grands classiques du XVII ${ }^{\mathrm{e}}$, l'abbé Gélinas fait connaître Louis Veuillot et Frédéric Mistral, le chantre du régionalisme

10. Nérée Beauchemin (1850-1931) passe sa vie à Yamachiche où il pratique la médecine tout en consacrant ses loisirs à la poésie. Son recueil, Les Floraisons matutinales paru en 1897, lui vaut un siège à la Société royale du Canada. Le second recueil, Patrie intime, est publié grâce à Gélinas et Tessier en 1928.

11. Louis-Delavoie Durand (1888-1965) est un Trifluvien de naissance qui fait ses études dans sa ville natale et à Charlottetown avant d'être admis au Barreau en 1915. Il pratique le journalisme et fonde, à Trois-Rivières, deux journaux qui ne connaitront qu'une brève existence. Il tente de se faire élire à Ottawa en 1930 et 1935, et à la mairie trifluvienne en 1937, trois échecs qui ne le découragent pas de mettre son éloquence légendaire au service de la petite patrie. Son succès, il le remporta comme président des fêtes du $350^{\bullet}$ anniversaire de Trois-Rivières en 1934.

12. Le Ralliement, 4,3 (juin 1937): 33-36.

13. Le Nouvelliste, 26 janvier 1927.

14. «Éducateur accompli, qui a voulu élever pour son pays des catholiques convaincus et des patriotes agissants», note Joseph Barnard, à l'occasion du décès de Gélinas, Le Bien public, 27 janvier 1927.

15. Omer Héroux, Le Bien public, $1^{\text {er }}$ février 1927. 
français. De ce dernier, il fait lire les Mémoires et récits afin de promouvoir l'attachement «au milieu et aux gens qui nous entourent». Les romans de son oncle Antoine Gérin-Lajoie (Jean Rivard), l'influence majeure de $\mathrm{M}^{\mathrm{gr}}$ Laflèche et celle sans doute aussi de $\mathrm{M}^{\mathrm{gr}}$ Denis Gérin, oncle maternel et curé nationaliste de Saint-Justin, ont joué à un titre ou à un autre dans l'expression du régionalisme de Gélinas. Ce dernier exercera à son tour une influence déterminante sur son élève Albert Tessier et incitera un vieil ami, le poète Nérée Beauchemin, à publier ses vers à saveur régionaliste.

Nérée Beauchemin mérite bien le titre de «poète régional» que $L e$ Nouvelliste, quotidien de Trois-Rivières, lui décerne en 1928 lorsque le barde de Yamachiche fait paraître Patrie intime à la Librairie d'action canadienne-française. Il avait cédé avec bonne grâce à l'abbé Gélinas qui le pressait de publier un recueil ${ }^{16}$. Les thèmes régionalistes transparaissent dans la majorité des poèmes rassemblés dans Patrie intime de même que l'idéalisation de la mère-patrie. Des titres comme «Ô patrie», «Notre terre», «Le laboureur», «Le ber», «Reste française», «Les lys», «La bonne France», sont évocateurs:

Et mon amour le plus pieux, / Et ma fête la plus fleurie, / Est d'avoir toujours sous les yeux / Le visage de ma patrie / Patrie intime de ma foi, / Dans une immuable assurance $[\ldots]^{17}$.

Le lien avec la France est souligné avec émotion:

Ma France, l'intime France, / C'est mon foyer, mon berceau, / C'est le lieu de ma naissance, / [...] C'est la terre où s'enracine l'Érable national ${ }^{18}$.»

Nérée Beauchemin se voit chargé d'honneurs à la sortie de son recueil. Célébration à l'hôtel de ville trifluvien, doctorat honorifique de l'Université Laval, médaille de l'Académie française et diplôme de «maître ès Jeux florimontains» soulignent son œuvre de poète régionaliste. Un disciple fervent avait orchestré cette gloire tardive, l'abbé Albert Tessier, celui qui prendra la relève après le décès de Gélinas en 1927 et de Beauchemin quatre ans plus tard.

Il faut remonter à la prime enfance d'Albert Tessier pour retracer les origines de sa vocation d'animateur et de régionaliste. Élevé en milieu rural, le jeune Tessier communie très tôt aux plaisirs

16. L'abbé Gélinas avait écrit un article sous le pseudonyme de Jacques Hertel, $L$ 'Écho de Saint-Justin, $1^{\text {er }}$ décembre 1923.

17. Armand Guillemette, Nérée Beauchemin. Son œuvre, édition critique (Montréal, Presses de l'Université du Québec, 1973 et 1974), 1: 149.

18. Ibid., 266. 
agrestes $^{19}$, les travaux de la ferme familiale lui procurant l'occasion de s'imprégner de la nature qu'il chérit. Au Séminaire de Trois-Rivières, Tessier a des maîtres qui lui donnent le goût de l'histoire et alimentent son nationalisme naissant dans le milieu où $M$ Laflèche avait imposé sa doctrine «nationale» et ultramontaine. Mais ce sera surtout l'abbé Gélinas qui affermira la vocation de Tessier. La lecture de Veuillot, de Mistral, l'évocation des hauts faits du passé, ceux du Régime français au premier chef où se distinguèrent plusieurs Trifluviens, ont fait du jeune Albert Tessier un enthousiaste de la petite patrie. Le voyage à Maillane, en Provence ${ }^{20}$, là où avait vécu Mistral, déterminera de façon définitive sa vocation régionaliste. Il succède à l'abbé Gélinas peu après son retour à Trois-Rivières, comme titulaire de la classe de rhétorique et préfet des études. Il se met à la rédaction d'articles en vue de préparer les esprits aux fêtes du Tricentenaire de la fondation de la cité de Laviolette. Il écrit par exemple, en 1928, que la Société d'histoire régionale veut réveiller «le sens régional, la fierté locale fondée sur l'attachement à un long et riche passé ${ }^{21}$ ». Le programme du régionalisme mauricien ne peut être mieux résumé que par cette formule.

Albert Tessier est convaincu que les moyens de diffusion que luimême met en œuvre - l'écrit, le reportage photographique et le film - stimuleront la fierté des Trifluviens en révélant «les grandeurs de leur histoire trois fois séculaire, le charme de leur terre aux lignes adoucies, la majesté impétueuse de leur triple rivière aux lourdes eaux bronzées $^{22}$ ». Pareil réveil doit déboucher sur l'action, et la célébration du tricentenaire constitue à ses yeux l'occasion rêvée d'une mobilisation des énergies. Selon lui, le régionalisme bien compris s'identifie à une pédagogie véritable. «Développer le sens de l'observation chez les jeunes; leur apprendre à voir plus loin que l'écorce, à pénétrer l'âme des choses [...] n'est-ce pas un peu du régionalisme ${ }^{23}$ ?», écritil à Olivar Asselin. Mais cette pédagogie ne rejoint pas que l'écolier. Toute la population est conviée à l'étude de son milieu, «les plantes,

19. Albert Tessier a évoqué sa jeunesse dans Souvenirs en vrac (Montréal, Boréal Express, 1975), autobiographie riche de témoignages captivants.

20. La quasi-totalité de la vie de Frédéric Mistral (1830-1914) se déroule en Provence dont il se fait le défenseur de la langue et de la culture. Auteur de Mireille, qui lui vaut le prix Nobel de littérature, Mistral est au cœur du renouveau occitan avec le mouvement félibre qu'il anime et la rédaction d'un monumental dictionnaire provençal-français.

21. Le Bien public, 25 octobre 1928. Le mot régionalisme paraît pour la première fois sous sa plume dans un article où il propose d'appliquer la doctrine de Mistral aux diverses collectivités régionales canadiennes-françaises. Voix régionales (1927): 355-358.

22. Le Bien public, 19 octobre 1933.

23. Lettre à Olivar Asselin, 2 avril 1933. Archives du Séminaire de Trois-Rivières (ASTR), Fonds Albert-Tessier, correspondance P-1. 
les bêtes, les travaux des hommes, les hommes eux-mêmes, ceux du présent d'abord, puis ceux du passé24 ». Ce n'est qu'une formule de départ: «À mesure que la vie et ses découvertes élargiront le cercle de nos activités, nous amplifierons au même rythme nos curiosités et nos désirs de mieux étudier [...] l'univers.» L'expression la plus souvent accolée à «régionalisme» est «bon sens», puis viennent les mots «réalisme» et «sain». Tessier affirmera plus tard que ce n'est pas le terme régionalisme qu'il aurait dû employer, mais «réalisme ${ }^{25}$ ». Cette nuance a sans doute été suscitée par des critiques à l'effet que ses idées pouvaient mener à l'étroitesse d'esprit, au chauvinisme. À un détracteur trifluvien, l'apôtre du régionalisme réplique sur un ton passionné: «Si le régionalisme est la fatuité puérile de gens qui ne voient rien de beau ni de bon en dehors de leur patelin, on a raison de crier haro sur ce baudet ${ }^{26 ! »}$

Par ailleurs, l'analyse de contenu des propos de Tessier a mis en relief plusieurs idées ou termes associés fréquemment à la notion de régionalisme. Curieusement, le mot nationalisme est rarement présent et, quand il paraît, le terme régionalisme (au sens de Tessier) peut lui être substitué ${ }^{27}$. En revanche, le régionalisme est rattaché au patriotisme à plusieurs reprises, le premier étant le préalable du second ${ }^{28}$. Par ailleurs, on ne s'étonnera guère de retrouver dans les propos d'un membre du clergé des thèmes de nature religieuse. Par exemple, il écrit que régionalisme «est tout simplement la mise en valeur aussi complète que possible de ce que Dieu a mis à la disposition de cha$\operatorname{cun}^{29} \gg$. Dans son optique, le régionalisme, acception particulière du patriotisme, est de nature essentiellement morale: «le patriotisme n'est pas une vertu facultative mais un mode de vie exigé de tous ${ }^{30}$ » comme, ajoute-t-il, l'enseigne saint Thomas d'Aquin qui établit un

24. Le Nouvelliste, 18 décembre 1936.

25. Albert Tessier, Pèlerinages dans le passé (Montréal, Fides, 1942), 16-18, et Le Devoir, 12 octobre 1946.

26. Le Flambeau, 1,1 (janvier-février-mars 1935): 13.

27. Ainsi à Henri Bourassa qui qualifiait ironiquement Trois-Rivières de «ville nationale par excellence» (voir Robert Rumilly, Henri Bourassa. La vie publique d'un grand Canadien (Montréal, Éditions Chanteclerc, 1953), 741), Tessier répond: «Notre nationalisme est calme, équilibré, appuyé sur les plus sûrs principes de la justice et de la charité [...] Réveiller le passé, prêcher la fierté, le culte du travail, la mise en valeur de nos richesses locales [...] il y a là [...] une formule d'action qui, en définitive, ne peut qu'enrichir la vie canadienne.» Le Flambeau, 2,5 (janvier-février-mars 1936): 5-6.

28. «Le régionaliste estime donc avec raison que tout effort tendant à nous plonger plus intensément dans la vie réelle, à nous enraciner dans notre milieu [...] est du patriotisme authentique.» Cité par Dauphin-Meunier, "Ceux qui firent notre pays», Études américaines (Paris, 1950).

29. Le Nouvelliste, 23 novembre 1940.

30. Le Bien public, 18 octobre 1934 et L'Action catholique, 7 novembre 1934. 
rapport indissoluble entre le patriotisme et la vertu de religion. La primauté du spirituel sur le temporel intervient à quelques reprises quoique de façon moins insistante que chez l'abbé Gélinas ${ }^{31}$.

Cependant, le régionalisme promu n'est pas pure spéculation. Il s'arrime à une vision du monde fondée sur l'observation de la réalité ambiante. «Développer le sens de l'observation ${ }^{32}$ », répète en effet l'animateur du régionalisme à tous les publics, jeunes et adultes, auxquels il s'adresse. Pour mener à bien une action constructive, il préconise l'utilisation systématique des moyens de diffusion: la presse, la photographie et le cinéma et veut même embrigader le théâtre et la radio ${ }^{33}$. En outre, le discours fait souvent référence au «concret», au «réalisme» et dénonce à maintes reprises le «théorique» et le «livresque». Par ailleurs, le véritable régionalisme est animé par les leçons de l'histoire. Albert Tessier met la main à la pâte et rédige de nombreux textes historiques, volumes, articles de périodiques et de journaux en plus des centaines de conférences qu'il prononce assisté de «projections lumineuses ${ }^{34} »$. Une véritable philosophie de l'histoire est énoncée: l'histoire doit réincarner le passé, devenir «communion», «atteindre et toucher les âmes» en présentant «des tranches de vie chaudes, colorées, palpitantes ${ }^{35}{ }^{\text {}}$. Autre composante du discours régionaliste mauricien: la Nature. Tessier prend les accents du poète quand vient le moment de célébrer l'environnement. En particulier, l'eau le fascine, comme le courant tumultueux du Saint-Maurice, source «du progrès matériel de la vallée du Saint-Maurice: métallurgie des Vieilles Forges, foyer de l'énergie électrique ${ }^{36}{ }_{\text {». }}$. Au thème de l'eau s'ajoute celui de la forêt mauricienne, symbole du Canada sauvage ${ }^{37}$.

Ces propos s'inscrivent dans une vision ruraliste de l'avenir de la société canadienne-française. «C'est le retour à la vie champêtre qui [...] favorisera le mieux la discipline d'âme», écrit Tessier dans la

31. Les Trifluviens ne doivent pas se laisser éblouir par les «transformations matérielles» amenées par l'industrialisation et la croissance démographique accélérées». Le Bien public, 25 octobre 1928.

32. Le Bien public, 2 mars 1933; Le Nouvelliste, 3 avril 1933.

33. L'Action nationale, 9,5 (mai 1937): 258-268.

34. Tessier produit une œuvre considérable qui s'étend de 1909 à 1975: 540 titres sont recensés pour la période finissant en 1946 dans la Bio-bibliographie de M. l'abbé Albert Tessier dressée par Lucienne Leduc. Parmi les volumes, mentionnons Trois-Rivières 1535-1935, Ceux qui firent notre pays, Pèlerinages dans le passé, L'énigme américaine, Les Forges du SaintMaurice et une Histoire du Canada en deux tomes. Ses productions cinématographiques atteignent la quinzaine et sont réalisées entre 1931 et 1949.

35. Voir notamment l'introduction de Pèlerinages dans le passé (Montréal, Fides, 1942), 9-18.

36. Le Bien public, 22 septembre 1932.

37. Voir l'introduction de Ceux qui firent notre pays (Montréal, Zodiaque, 1936), 14-19. 
conclusion de Ceux qui firent notre pays. En 1957, il s'opposera à Marcel Trudel, lequel affirmait que Champlain avait fondé la colonie dans un but mercantile ${ }^{38}$. Par ailleurs, l'industrialisation est pointée du doigt lorsque vient le moment pour Tessier d'identifier les causes de la crise économique: «un développement trop rapide a empêché de suivre le processus normal de la nature ${ }^{39}$ », précise-t-il en 1933. Il est également sensible à l'urbanisation accélérée et chaotique de la Mauricie car, aux «cubes de briques que les escaliers extérieurs font ressembler à des cages à poules», il préfère le charme vieillot des vieilles demeures «normandes ${ }^{40}$ ». Malgré la lutte menée contre les affiches unilingues anglaises, Tessier se réjouit du tourisme non seulement «étranger» mais «intérieur»: les Mauriciens doivent visiter leur coin de pays en premier lieu.

Le propagandiste du régionalisme mauricien a insisté sur l'importance du développement de la personnalité chez l'individu ${ }^{41}$. Éducateur de carrière qu'il était, il ne pouvait passer sous silence l'importance de la formation des jeunes. Il parle d'un «capitaljeunesse» pour Trois-Rivières qui compte $46 \%$ de sa population en bas de 20 ans au recensement fédéral de 1931. Il en tire la conséquence qu' «il y a là des ressources formidables, pour le bien ou pour le mal, selon le soin ou la négligence qu'on apportera à former cette jeunesse ${ }^{42}$ ». Par ailleurs, l'expression «race française» ou «race canadienne-française» revient peu souvent même si la «ténacité nordique» des Trifluviens, fils de Percherons et de Normands, est évoquée dans une lettre à Olivar Asselin ${ }^{43}$. Finalement, Albert Tessier se garde bien de parler politique, mais il s'épanche longuement sur la mission d'un État idéal ébauchant ainsi une véritable utopie qui paraît en $1937^{44}$. Le rôle de l'État est de direction spirituelle, affirme-t-il, ajoutant que le grand œuvre de Mussolini consiste dans «la rénovation même du peuple italien ${ }^{45}$ ». À cette fin de rénovation, Tessier propose la création d'une commission d'éducation nationale dont le mandat serait d'atteindre «la masse et lui façonn[er] une mentalité plus ouverte, plus éveillée, plus consciente» afin de «retremper l'âme

38. Dans l'article «France nouvelle ou simple colonie commerciale?», Cahiers des Dix, 22 (1957): 43-51 où il présente un compte rendu critique du Champlain de Trudel (Montréal, Fides, 1956).

39. Le Nouvelliste, 3 avril 1933.

40. Le Bien public, 2 mars 1933.

41. «Le régionalisme vivant [...] est basé sur le perfectionnement de soi-même», Ibid.

42. Trois-Rivières 1535-1935 (Trois-Rivières, Le Nouvelliste, 1935), 187.

43. Lettre à Olivar Asselin, 2 avril 1933, ASTR, Fonds Albert-Tessier, correspondance P-1.

44. «Pour une politique nationale», L'Action nationale, 9,5 (mai 1937): 258-268.

45. Loc. cit., 130. 
canadienne-française, moyen le plus direct d'éveiller l'amour de la Patrie». En résumé, le thème régionaliste forme le noyau du discours social d'Albert Tessier énoncé entre 1927 et 1940. Il fait du culte de la petite patrie l'étape initiale et indispensable de toute perception du monde ancrée dans le réel. À leur tour, les propos régionalisants de Louis-Delavoie Durand s'inscrivent dans une perspective concrète de la vie sociale.

Durand est avant tout un nationaliste ardent. Il rédige des textes vibrants dans Le Devoir, L'Action française de Montréal et L'Assaut de Paris. Son admiration va à Maurice Barrès, Charles Maurras et surtout Lionel Groulx. Son nationalisme a de particulier qu'il s'enracine dans l'amour du coin de pays natal. Orateur apprécié, LouisDelavoie Durand multiplie les conférences et les causeries radiophoniques où le thème du régionalisme est omniprésent. Choisi président des fêtes du Tricentenaire, il traduit par l'action son amour de la petite patrie et, dans ses propos, il n'hésite pas à avancer des arguments d'ordre moral et économique. En temps de crise, affirme$\mathrm{t}$-il, il faut donner une nouvelle vigueur morale aux gens «en illustrant le labeur des ancêtres, [...] en prenant conscience de notre destinée, en affirmant avec la dignité de notre passé nos droits d'aînesse ${ }^{46}{ }$. Dans sa perspective, le régionalisme naît d'un «besoin d'économie. On l'institue afin que rien ne soit perdu des activités et des possibilités d'une région ${ }^{47}$ ». Pour lui, le régionalisme repose sur «l'instauration d'une vie de famille entre les membres divers d'une même région, qui donne à chacun l'orgueil de sa petite patrie [...], c'est une attitude devant la vie, idéaliste et réaliste à la fois ${ }^{48} \gg$. En outre, Durand défend l'esprit «bourgeois» fondé sur l'ordre, l'économie, la vie de famille qui constitue le fondement de «sa vie raciale». Le tribun trifluvien donne ce qui lui apparaît comme le sens vrai des efforts régionalistes:

Or la région, la Mauricie, c'est ce qu'il y a de plus solide parce que cela implique ce qui seul demeure et que nous aimons: le clocher, le jardin, le cimetière, les trois pôles qui, nous retenant le mieux à la terre de chez nous, nous empêcheront le mieux de continuer en série la fabrication de nomades [sic] de l'asphalte.

Durand développe un programme économique à l'occasion de ses campagnes à la mairie et aux élections fédérales. Une perception de l'espace régional englobant la rive sud jusqu'à Victoriaville et

46. Le Nouvelliste, 4 avril 1933. L'article reproduit le mémoire présenté par Durand au Conseil des ministres à Québec dans le but d'obtenir une aide financière pour les fêtes du Tricentenaire.

47. L'Almanach trifluvien, 4 (1935): 86.

48. Le Nouvelliste, 28 janvier 1936. 
Drummondville lui inspire l'idée d'une voie de communication franchissant le fleuve devant Trois-Rivières. Il insiste dans sa publicité électorale sur le caractère essentiel de l'expansion industrielle et commerciale de la Mauricie et de l'aménagement de voies de communication rapides qui contribueront au succès du développement régiona ${ }^{49}$. On notera, par ailleurs, que son discours régionaliste ne présente pas de références religieuses, sauf rare exception. Son discours se situe donc dans la foulée des libéraux étudiés par Fernande Roy ${ }^{50}$.

En somme, la triade du régionalisme mauricien: amour de la petite patrie, culte des héros du passé et valorisation de la vie rurale, trouve son écho dans le discours de Durand mais avec une coloration différente, affichant moins vigoureusement les idées clérico-nationalistes et plus ouvertement les valeurs du libéralisme économique.

Comment soupeser le rôle respectif de chacun des quatre acteurs principaux et spécifier la pensée sociale qui a étayé leur discours? En bref, on peut affirmer que Gélinas a été le précurseur immédiat en posant les fondements idéologiques du discours; Beauchemin les a illustrés par les sortilèges de la poésie. Tessier, quant à lui, qui a donné un nom à cette doctrine, le régionalisme, l'a humanisée et, surtout, l'a diffusée dans toutes les couches de la population. Enfin, Durand a ajouté une touche économique et politique sans toutefois exercer d'influence déterminante dans le contenu. Au thème platonicien de l'Idéal chez Gélinas, Tessier et Durand pourvoient l'imaginaire mauricien d'une symbolique tirée de la nature sensible: métaphore de la famille chez Durand, symbolique de l'eau vive chez Tessier.

Quoique étant de nature foncièrement spéculative, leurs propos n'ont pas moins donné l'impulsion à des actions collectives capables de provoquer des changements d'ordre culturel ou social en s'insérant dans la dynamique régionale. Les intellectuels trifluviens se sont mobilisés dans des activités littéraires et historiques, ont participé aux fêtes du Tricentenaire, milité au sein de la Société Le Flambeau et du Syndicat d'initiative ou encore «ferraillé» dans le débat sur le terme «Mauricie». Chacun de ces modes d'engagement social doit être abordé séparément afin de saisir le mouvement régionaliste dans sa globalité.

49. Le projet d'un musée régionaliste revient à plusieurs reprises mais, comme le projet du tunnel, ne se réalisera jamais.

50. Fernande Roy, Progrès, harmonie, liberté. Le libéralisme des milieux d'affaires francophones à Montréal au tournant du siècle (Montréal, Boréal, 1988), $301 \mathrm{p}$. 


\section{3 - LE RÉGIONALISME MAURICIEN ET LA SOCIÉTÉ RÉGIONALE}

Le débat sur le régionyme Mauricie a soulevé les passions durant plusieurs mois en 1933 et en 1934. Albert Tessier s'est tout naturellement retrouvé au cœur des discussions puisque c'est à lui que revient la paternité du nouveau mot ${ }^{51}$. Peu après son retour d'Europe, Tessier s'emploie à trouver une alternative à «vallée du Saint-Maurice ${ }^{52}$ » et utilise, pour la première fois publiquement, «Mauricie» en octobre $1933^{53}$. Le nouveau régionyme semble faire l'unanimité tout d'abord, mais une opposition se manifeste de plus en plus vigoureusement dans la presse locale; les partisans de Mauricie répliquent avec ardeur: c'est que «Mauricie» avait eu l'heur de déplaire à l'évêque auxiliaire de Trois-Rivières, $\mathrm{M}^{\mathrm{gr}}$ Alfred-Odilon Comtois. Ce dernier fustige publiquement les tenants du nouveau mot en risquant un calembour de son cru: «Jusqu'ici je croyais vivre dans la région du Saint-Maurice. Les historiens m'apprennent que je vis dans la Mauricie. Non, pas la mort $i c i^{54}$ !» Poursuivant son algarade, le prélat allègue que «Mauricie» enlève à Trois-Rivières sa primauté sur l'arrière-pays, et propose «Trifluvianie ${ }^{55}$ ». Quelques membres du clergé soutiennent l'évêque dans des lettres envoyées aux journaux trifluviens ${ }^{56}$; ils reçoivent l'appui de plusieurs anglophone $\mathrm{s}^{57} \mathrm{craignant}$ que les touristes ne soient déroutés. Plusieurs journaux de la région et aussi de Montréal font écho à la dispute ${ }^{58}$; finalement, les esprits s'apaisent, le néonyme s'impose sans heurt. Mais le débat se ranime trois ans plus tard quand

51. Avant 1850, l'expression «district de Trois-Rivières» est la plus courante pour désigner le territoire couvrant l'ancien gouvernement des Trois-Rivières du Régime français, comme l'attestent les documents officiels et les journaux de l'époque. Le district judiciaire de Trois-Rivières avait été créé à la fin du XVIII ${ }^{e}$ siècle. Cette désignation recule devant «vallée du Saint-Maurice», calque de St.Maurice Valley, qui s'impose peu à peu avec l'exploitation forestière du bassin de la rivière Saint-Maurice.

52. Il songe d'abord à «Bouches-du-Saint-Maurice», inspiré de «Bouches-du-Rhône», puis à «Bouches du Métabéroutin» (nom amérindien du Saint-Maurice) avant de fixer son choix sur «Mauricie», calquée sur «Laurentie».

53. À l'occasion du banquet d'inauguration du Syndicat d'initiative, il venait de changer le titre d'un manuscrit sur l'histoire de La Tuque, qui lui avait été soumis par Lucien Desbiens. Au portique des Laurentides avait été remplacé par Au cœur de la Mauricie, ASTR, Fonds Albert-Tessier, Q2-71.

54. Tel que le rapporte Tessier lui-même dans Souvenirs en vrac, 166.

55. Se rappelant la division douloureuse du diocèse en 1885 (alors que $\mathbf{M}^{\mathrm{gr}}$ Laflèche avait perdu Nicolet et toute la rive sud), $M^{8 r}$ Comtois aurait-il craint la création d'un nouveau diocèse à Shawinigan à la suite de la croissance démographique rapide? L'hypothèse mériterait à notre sens d'être vérifiée.

56. Le Nouvelliste, 3 et 5 janvier 1934; Le Bien public, 4 janvier 1934.

57. St.Maurice Valley Chronicle, 11 janvier 1934 et $1^{\text {er }}$ février 1934.

58. Notamment La Chronique de la Vallée du Saint-Maurice, L'Écho du Saint-Maurice, La Gazette du Nord, L'Action catholique, Le Devoir, et Le Petit journal. 
Henri Bourassa, prononçant une conférence à Sherbrooke, s'emporte contre certains jeunes gens désœuvrés qui «inventent la Laurentie, la Mauricie, toutes sortes de 'scies ${ }^{59}$ ' . Il n'en fallait pas plus pour soulever l'ire d'Albert Tessier qui riposte vertement ${ }^{60}$. Après, tout rentre dans l'ordre et la Mauricie continuera de désigner la région trifluvienne jusqu'à notre époque.

On peut être étonné de l'ampleur prise par la question; toutefois, la compréhension des enjeux symboliques en présence fait ressortir l'importance de l'événement. La coïncidence entre l'éveil de la conscience régionale et la création d'un nouveau régionyme n'est pas le fait du hasard: après avoir bâti le pays, il faut le nommer. Une innovation toponymique consacrait dans l'ordre du discours la réalité des changements sociaux qui avaient contribué à la mise en place d'une conscience de la région. Le terme «Mauricie» prenait une connotation positive aux oreilles de ceux qui envisageaient l'avenir avec optimisme et une allure mortifère pour les nostalgiques du XIX ${ }^{\mathrm{e}}$ siècle agricole et «paisible». Mais l'unanimité totale se fera quand viendra le moment d'organiser les fêtes du Tricentenaire de Trois-Rivières, au printemps 1934.

Les fêtes commémorant le $300^{\mathrm{e}}$ anniversaire de la fondation de Trois-Rivières par Laviolette le 4 juillet 1634 ont constitué l'illustration la plus visible de l'action régionaliste ${ }^{61}$. L'idée des fêtes remonte à 1926, année à partir de laquelle les publications de la Société d'histoire régionale paraissent à une cadence rapide en vue de préparer les esprits. En revanche, le contenu des célébrations tarde à se préciser; s'agira-t-il d'une exposition-musée, de la reconstitution du vieux fort ou d'un «pageant» historique? Ce n'est qu'au dernier moment, soit en avril, deux mois avant la date prévue ${ }^{62}$, que les responsables arrêtent la formule définitive: un ensemble de célébrations religieuses ou historiques alternant avec des festivités populaires susceptibles d'attirer le grand public.

59. La Tribune, 23 janvier 1937. La charge de Bourassa se comprend dans l'opposition qu'il manifeste à Laurendeau qui reprend le terme Laurentie en 1935 (Notre nationalisme, brochure des Jeunes-Canada).

60. Sous le pseudonyme de «Le Tisserand», Tessier qualifie Bourassa de sénile (Le Bien public, 11 février 1937). Un journaliste du Canada qui appuie Bourassa prend ironiquement le pseudonyme d'Hochelagacien et attaque Le Bien public, l'associant à l'Union nationale de Duplessis.

61. Albert Tessier affirme sans hésiter que l'événement fut «à la base du mouvement régionaliste». Le Bien public, 12 mars 1935.

62. Un comité d'initiative formé d'élus municipaux, de membres de la Chambre de commerce, de la Commission du Hâvre et de la Société d'histoire régionale multiplie les demandes d'aide financière auprès d'Ottawa et de Québec. Tessier enjoint Duplessis de lui venir en aide dans une lettre confidentielle datée du 24 juin 1933, ASTR, Fonds Albert-Tessier, correspondance, P-1. 
Reconstitutions historiques, défilés en costumes, dévoilements de monuments, discours patriotiques se succèdent sans relâche, mais le clou des manifestations est sans conteste les représentations des «pageants» avec le concours de 500 figurants et comédiens volontaires $^{63}$, recrutés chez la jeunesse trifluvienne. Une suite de 22 «tableaux» proposent une synthèse de l'histoire de Trois-Rivières depuis les débuts en mettant fortement l'accent sur le Régime français. L'action dramatique est soutenue par une imposante chorale et la Philharmonie De-La-Salle. Trois représentations avaient été prévues: devant l'accueil enthousiaste du public, sept autres leur seront ajoutées. Le déplacement des troubadours en costume dans les rues, les vendeuses de magasin déguisées en Bretonnes du XVII ${ }^{\mathrm{e}}$, les banderolles, drapeaux et écussons contribuent à créer une évasion dans le passé. Trois-Rivières transformée accueille de nombreux touristes auxquels s'ajoutent les participants aux congrès et ralliements qui ont profité de l'occasion pour se réunir dans la capitale mauricienne.

L'esprit de ces fêtes épouse les contours du discours régionaliste. Cette concordance n'a rien de surprenant, les deux principaux instigateurs des célébrations, Albert Tessier et Louis-D. Durand ne sont-ils pas justement les porte-parole majeurs du régionalisme à Trois-Rivières? La glorification des héros locaux du Régime français ${ }^{64}$ représente le lien le plus manifeste entre le mouvement de fierté régionale et la reconstitution du passé à laquelle l'été 1934 a donné lieu $^{65}$. Véritables stratégies d'éducation et d'intégration aux valeurs promues par la petite bourgeoisie locale, les «pageants» et autres manifestations populaires ont contribué habilement à l'affermissement d'une conscience régionale bien encadrée dans le schème clérical et nationaliste des années trente au Canada français. Habilement, car les organisateurs avaient dû faire appel non seulement à l'intellect, mais aux sentiments et aux pulsions nées de l'ivresse de la fête. Il y aurait d'ailleurs des similitudes à relever avec les splendeurs de la liturgie catholique qui cherche à rejoindre les fidèles par toutes les fibres de leur sensibilité ${ }^{66}$.

63. Pour le contenu des «pageants», leur signification symbolique et le rôle qu'ils ont joué dans le mouvement trifluvien, voir Rémi Tourangeau, Trois-Rivières en liesse: aperçu historique des fêtes du Tricentenaire (Trois-Rivières, CEDOLEQ, 1984).

64. Notamment les Lavérendrye, Radisson, Jacques Hertel, Jean Godefroy, Jean Nicolet et plusieurs autres.

65. L'entreprise idéologique sous-jacente aux Fêtes a réussi pleinement si l'on en juge le témoignage des auteurs de l'époque. Voir Rémi Tourangeau, op. cit., 157-158, et le témoignage de Clément Marchand à l'auteur, entrevue du 12 avril 1988.

66. Les fêtes religieuses associées au Congrès eucharistique de Trois-Rivières tenu sept ans plus tard ont revêtu un éclat tout à fait comparable à celles de 1934. Voir Mémorial du Premier Congrès eucharistique diocésain tenu aux Trois-Rivières du 20 au 24 août 194I (TroisRivières, s.é., 1942). Une partie importante des textes et des photographies sont d'Albert Tessier. 
Les activités de la Société Le Flambeau s'inscrivent, elles aussi, dans le courant régionaliste d'une façon explicite. Mais cette fois ni Tessier $^{67}$ ni Durand ne figurent au premier plan même si leur appui ne s'est pas démenti. L'idée de rassembler la jeunesse trifluvienne afin de prendre part à l'organisation des fêtes du Tricentenaire prend corps au début de 1934. De jeunes professionnels, des anciens du Séminaire Saint-Joseph et des étudiantes des Ursulines se réunissent afin de mettre sur pied une association ayant comme objectifs de promouvoir les recherches historiques, encourager le développement des arts, l'étude des sciences et la connaissance des lettres et, enfin, aider les mouvements de fierté trifluvienne ${ }^{68}$. La Société emprunte son nom au projet qu'elle met de l'avant, soit l'érection d'un monument représentant un flambeau porteur de la flamme, symbole de l'idéal moteur de la jeunesse trifluvienne. Les enfants de toutes les écoles et les jeunes ouvriers contribuent à une souscription publique menée avec ferveur ${ }^{69}$. Le député de Trois-Rivières aux Communes, Charles Bourgeois, dont la fille siège sur le comité, réussit à obtenir $7000 \$$ du ministère Bennett. Le Flambeau est inauguré le 12 août à l'occasion d'une fête grandiose où s'anime une chorale de 700 voix devant une assistance composée de milliers de jeunes. La Société Le Flambeau n'en reste pas là. Les responsables concrétisent un autre élément de leur programme en publiant un périodique intellectuel, Le Flambeau. Cinq numéros paraissent en 1935 et 1936. En tête de la première livraison, le directeur Louis-Philippe Poisson écrit que la symbolique du flambeau crée une «mystique trifluvienne»; il précise que la revue ne verra pas dans le régionalisme une fin en soi, mais l'élément d'une saine éducation afin de «constituer un idéal national assez fort pour s'imposer et inspirer confiance en l'avenir ${ }^{70} \gg$. Les sujets abordés sont les héros de l'histoire, la confiance en l'avenir, l'action soutenue par un idéal spirituel, l'oubli de soi dans une cause collective et le développement de la personnalité. On note que l'exaltation de la nature et de la vie rurale est mise de côté et que la «reconquête» envisagée n'est plus seulement d'ordre moral ou spirituel, mais économique et politique $^{71}$. Ainsi, la fierté n'est plus établie seulement sur les promesses du passé, mais actualisée dans le développement personnel et le refus du repli. Ces propos novateurs pour l'époque écartent quelque peu

67. Malgré ce qu'a écrit Louis Caron dans La Tuque et le bérêt (Montréal, L’Archipel/ Édipresse, 1992).

68. Le Nouvelliste, 8 mars 1934.

69. Une somme de $1212,25 \$$ est rassemblée en trois mois.

70. Le Flambeau, 1,1 (janvier-février-mars 1935): 3.

71. La séparation éventuelle de la Province hors de la Confédération est même évoquée. Voir l'article d'Hervé Biron, Le Flambeau, 1,3 (juillet-août-septembre 1935): 60. 
l'idéologie du Flambeau de celle de Tessier et de Gélinas, mais pas de façon assez intense pour mener à une rupture.

Le régionalisme des années trente a marqué d'une empreinte profonde la vie culturelle trifluvienne. Un noyau de clercs et de laïcs s'emploient à promouvoir l'histoire locale, la littérature canadiennefrançaise et aussi une conception bien particulière de la culture française. Fondée en $1926^{72}$, la Société d'histoire régionale organise des conférences et des pèlerinages historiques ${ }^{73}$; elle est à l'origine de deux collections de monographies historiques, «Les Cahiers d'histoire régionale» et «Les Pages trifluviennes». La première série se poursuivra jusqu'aux années cinquante avec des travaux entre autres de Raymond Douville et d'Albert Tessier, et la seconde comprendra 36 plaquettes parues entre 1932 et 1939 mobilisant les efforts des historiens locaux. Le quotidien Le Nouvelliste, qui commence à paraître en 1920, emboîte le pas à l'effort régionaliste ${ }^{74}$, mais c'est surtout Le Bien public ${ }^{75}$ qui s'identifie au programme de Tessier. Deux jeunes journalistes se voient confier la direction du Bien public grâce à l'intervention d'Albert Tessier. Clément Marchand et Raymond Douville se font les propagandistes enthousiastes du réveil mauricien, le premier sous l'angle littéraire, le second dans une optique historique. Trois périodiques ont également exercé une influence certaine durant cette riche décennie. Outre Le Flambeau, cité précédemment, L'Almanach trifluvien (1932-1935) et Le Mauricien (19361939) servent de tribune à Tessier, Marchand, Durand et Douville. L'Almanach trifluvien prend modèle sur L'Almanach provençal de Frédéric Mistral en ouvrant largement ses colonnes à la littérature et à l'histoire; Le Mauricien se présente comme une «revue régionaliste illustrée» dans le genre de La Revue populaire. Il publie chaque mois des textes d'auteurs trifluviens, mais accorde une large place à des textes inédits d'auteurs de Montréal et de Québec ${ }^{76}$.

Dans la Mauricie de 1930-1940, nombre de productions littéraires doivent leur cachet particulier, sinon leur existence, aux mots d'ordre lancés par les régionalistes trifluviens. Un survol même rapide fait

72. Par des prêtres du Séminaire et des notables férus de petite histoire. Albert Tessier est élu secrétaire et joue un rôle de premier plan.

73. Notamment aux Forges de Saint-Maurice où Lionel Groulx et Louis-D. Durand prononcent des allocutions, et à Sainte-Anne-de-la-Pérade au pays de Madeleine de Verchères et de $\mathrm{M}^{\mathrm{gr}}$ Laflèche.

74. Avec des articles d'Hervé Biron et d'Yvon Thériault.

75. Fondé en 1909 par l'évêque de Trois-Rivières, Le Bien public diffuse l'idéologie clérico-nationaliste comme L'Action catholique s'inspire de Mgr Laflèche et appuie l'émergence du syndicalisme catholique.

76. Paraissent des récits ou poèmes de Louis Dantin, Damase Potvin, Rex Desmarchais, Harry Barnard, Alfred Desrochers, François Hertel, Robert Choquette et plusieurs autres. 
ressortir que la littérature mauricienne s'inscrit alors dans le projet du régionalisme littéraire en bonne partie. C'est que Tessier avait incité les écrivains locaux à exploiter la richesse que constitue la Nature, à promouvoir l'effort tenace et illustrer l'amour du risque dans leurs récits et leurs poèmes ${ }^{77}$. Clément Marchand, qui avait pris la direction du Mauricien avec Douville en 1937, faisait de son côté l'apologie d'un régionalisme plus ouvert à la réalité urbaine et d'une littérature canadienne modelée sur les écrivains célébrés par la droite française catholique ou régionaliste ${ }^{78}$. Plusieurs auteurs de la région répondent à ces appels empressés et, parmi ces auteurs, plusieurs femmes. Moïsette Olier ${ }^{79}$ publie d'abord L'Homme à la physionomie macabre en 1927; enthousiasmé par l'ouvrage, Tessier le qualifie de roman «régional». Paraissent ensuite Le Saint-Maurice ${ }^{80}$, hymne à la rivière Cha8inigane ${ }^{81}$ et histoire de la Mauricie en épopée, Étincelles ${ }^{82}$, évocation des Forges de Saint-Maurice, et Mademoiselle Sérénitế83 dont l'intrigue se noue dans l'atmosphère des fêtes du Tricentenaire. Jeanne L'Archevêque-Duguay ${ }^{84}$ fait paraître $E_{c} c^{2}{ }^{85}$, recueil de textes en vers et en prose illustrant des personnages trifluviens et la défense de la vie rurale. En outre, deux romancières de l'étranger, l'une française, Marie Le Franc ${ }^{86}$, et l'autre américaine, Sarah Larkin ${ }^{87}$, se sont attiré l'admiration des régionalistes en chantant la forêt mauricienne $^{88}$. De leur côté, La Rue des Forges ${ }^{89}$ de Phyl Laferrière ${ }^{90}$, Mon

77. Tessier, en réponse à des critiques, se défend de confondre régionalisme et terroirisme. Voir la lettre du 2 avril 1933 à Jean-Charles Harvey et celle du 3 avril 1933 à Olivar Asselin, ASTR, Fonds Albert-Tessier, correspondance P-1 et P-2.

78. Comme Francis Jammes, René Bazin, Henriette Charasson, Léon Daudet et Henri

79. Pseudonyme de Corinne P.-Beauchemin (1885-1972). L'influence de Tessier est soulignée dans l'étude que lui a consacrée Suzanne Lafrenière, Moïsette Olier: femme de lettres de la Mauricie (Ottawa, Asticou, 1980), 24-25 et 33-43.

80. Première partie d'Au pays de l'énergie (Trois-Rivières, Le Bien public, 1932).

81. Cha8inigane (Trois-Rivières, Le Bien public, 1934).

82. Étincelles (Trois-Rivières, Le Nouvelliste, 1936).

83. Mademoiselle Sérénité (Trois-Rivières, Le Nouvelliste, 1936).

84. Née en 1901, elle épousa le peintre Rodolphe Duguay. En plus de son œuvre poétique, elle a rédigé de nombreux articles dans des périodiques.

85. Écrin (Trois-Rivières, Le Bien public, 1934).

86. Marie Le Franc (1879-1964) publie en 1936 La Randonnée passionnée. «Le SaintMaurice fait son entrée dans la littérature française», écrit Raymond Douville, Le Flambeau, 1,3 (juillet 1935): 70.

87. Sarah Larkin (1896-1988) écrit un poème Three Rivers (1934) et un roman, Radisson (1938) inspirés de ses séjours en Haute-Mauricie.

88. Tessier, Marchand et Douville ont également salué un roman de Maurice Genevoix (1890-1980), Laframboise et Bellehumeur qui met en scène des trappeurs mauriciens. Genevoix avait visité la Mauricie en 1939 avec Albert Tessier comme guide.

89. La Rue des Forges (Montréal, Albert Lévesque, 1932).

90. Mon petit pays (Trois-Rivières, Le Bien public, 1932) et En flânant dans les portages (Trois-Rivières, Le Bien public, 1933). Sylvain est le pseudonyme du Dr Auguste Panneton, frère de Ringuet. 
petit pays et En flânant dans les portages de Sylvain s'inscrivent dans la thématique régionaliste par leur sujet. En revanche, Les Soirs rouges $^{91}$ de Clément Marchand et Trente arpents ${ }^{92}$ de Ringuet se situent dans un courant plus universaliste de la littérature. Le premier ouvrage, un recueil de poèmes, inaugure la thématique urbaine dans la poésie canadienne-française et le second, un roman d'abord publié à Paris, évoque la vie rurale mais sans complaisance aucune. Autre évocation de la misère urbaine, le recueil d'Alphonse Piché, Ballades de la petite extrace ${ }^{93}$, est à cent lieues du programme régionaliste de Tessier ${ }^{94}$. En bref, une vie littéraire et artistique intense a caractérisé la Mauricie des années trente. Les œuvres entraînées dans le sillon du régionalisme n'ont guère résisté à l'épreuve du temps. Par contre, les ouvrages de Marchand, Ringuet et Piché ont survécu, libérés d'une vision passéiste et d'un refus de la modernité. Il ne faut pas croire que les écrits régionalistes ont reçu un accueil unanimement favorable au moment de leur parution. Des distinctions sont nécessaires afin de nuancer la réception de l'œuvre régionaliste mauricienne. Effectivement, on constate que ce sont les intellectuels sollicités par Tessier, comme Benoît Brouillette et Raymond Tanghe, auxquels on peut ajouter Olivar Asselin et Jean-Charles Harvey, qui manifestent le plus de résistance. Seul fait exception Raoul Blanchard, dont la conception particulière de la géographie physique (et sans doute une amitié sincère qui le lie à Tessier) permet un rapprochement agissant.

L'Église a donné son accord à l'expression de ce qu'elle considérait comme une application particulière de la vertu de patriotisme définie par saint Thomas d'Aquin. Cette argumentation théologique légitime en fait la sympathie envers un mouvement issu du clériconationalisme et de l'idéologie du retour à la terre. Le rôle joué par le Séminaire Saint-Joseph dénote jusqu'à quel point la principale maison

91. Les soirs rouges (Trois-Rivières, Le Bien Public, 1947). Les poèmes qui composent le recueil avaient d'abord paru dans Le Bien public durant les années trente. Clément Marchand, né en 1912, a publié en outre Courriers des villages (Trois-Rivières, Le Bien public, 1942), qui offre une vision distanciée de la vie rurale.

92. Trente arpents (Paris, Flammarion, 1938). Ringuet est le pseudonyme du Dr Philippe Panneton (1895-1960). Il a signé plusieurs autres romans, comme Fausse monnaie (1947) et Le poids du jour (1949) et un ouvrage historique, Un monde était leur empire (1943). Trente arpents a été traduit en anglais et en allemand.

93. Ballades de la petite extrace (Montréal), Fernand Pilon, 1946). La date de la rédaction se situe en 1939. Né en 1917, Alphonse Piché a rassemblé son œuvre poétique en plusieurs recueils, de Remous (1947) à Néant fraternel (1991).

94. D'autres activités artistiques trifluviennes de 1930 à 1940 relèvent du courant régionaliste ou ont été appuyées par ses promoteurs, notamment la musique (J.-A. Thompson), la photographie (H. Rivard et J.-A. Denoncourt), les arts plastiques (J.-J. Spénard, H. Beaulac, L. Arbour) et la peinture représentée par Rodolphe Duguay (1891-1973) qui a exprimé la sérénité campagnarde dans ses paysages et ses gravures sur bois. 
d'enseignement à Trois-Rivières constituait un lieu d'énonciation idéologique dominant.

Les articles relatifs aux réalisations régionalistes de la Mauricie des années trente peuvent être regroupés en deux séries. La première insiste sur la description des événements, comme le Tricentenaire, et fait état des impressions immédiates des gens, alors que la seconde expose, justifie ou condamne la doctrine de l'école régionaliste. Les textes des commentateurs français et américains sont de nature anecdotique et présentent une élaboration littéraire qui excite la curiosité du lecteur. Cette approche vise peut-être moins la valeur intrinsèque du courant régionaliste que les circonstances qui ont mis les auteurs en contact avec ses réalisations. Les auteurs n'ont guère eu le temps d'appréhender le mouvement d'idées qui sous-tendait ces réalisations dont le caractère pittoresque a retenu leur attention. Toutefois, la majorité des articles se prononcent: soit qu'ils appuient l'école régionaliste, soit qu'ils la dénoncent. Une appréciation passionnée envers Albert Tessier, dont le charisme d'animateur est mis en évidence, est souvent présente. Il est d'ailleurs souvent malaisé de départager ce qui relève de l'adhésion logique ou de la fascination exercée par l'enthousiasme de Tessier.

L'impact du régionalisme mauricien s'est diversement traduit selon l'origine géographique et l'horizon culturel des récepteurs. La presse trifluvienne est presque unanimement dithyrambique, emportée qu'elle est par la fièvre du Tricentenaire et par la ferveur contagieuse d'Albert Tessier et de Louis-D. Durand. Les anglophones trifluviens ont réagi positivement, soulagés du fait que l'ordre social, loin d'être remis en question, recevait un appui collectif. L'accueil de la presse canadienne est plus nuancé. Les journaux et périodiques nationalistes ajoutent leur voix à la chorale régionaliste. Le Devoir, Le Droit, L'Action catholique et L'Action nationale n'ont que des éloges à l'endroit du régionalisme mauricien. Toutefois, les journalistes montréalais Olivar Asselin, Albert Pelletier et Jean-Charles Harvey résistent aux sirènes régionalistes en stigmatisant la naïveté, l'étroitesse de vue et l'attitude de repli que cette vision des choses reflète à leurs yeux. Par ailleurs, la compréhension de la presse étrangère ne dépasse pas une certaine curiosité envers un exotisme nostalgique. Ainsi, la réception au discours régionaliste varie selon que le récepteur est ou non un Mauricien, selon qu'il est nationaliste traditionnel ou non. Pour les uns, le régionalisme de Tessier se présente comme une vérité évidente; pour les autres, il représentait déjà une vision passéiste susceptible de mener au repliement. 


\section{4 - L'APPROPRIATION SYMBOLIQUE D'UN ESPACE RÉGIONAL}

Les intellectuels trifluviens cultivent la nostalgie de la mèrepatrie, non pas celle, réelle, du XX $\mathrm{XX}^{\mathrm{e}}$ siècle, mais la France de l'Ancien Régime, une France mythique, royale et catholique où, selon eux, l'harmonie avait régné entre les classes de la société, unies dans un corporatisme idéalisé. Beaucoup d'idéal et de générosité transpiraient de cette vision, mais elle correspondait de moins en moins à la réalité sociale en mouvement. Source première de ce clivage, le contexte de tension fondé sur l'appréhension des mutations sociales sera particulièrement exacerbé par la Grande Dépression. Il émerge donc une appétence idéologique nourrie par la recherche anxieuse d'une vérité amenant les réponses toutes faites aux problèmes du temps présent.

Nous croyons que l'impuissance à gérer les biens matériels alimente parfois le besoin de la gestion du symbolique, et nous en avons un exemple ici. Le régionalisme mauricien promeut des valeurs, tels la fierté, l'idéal agissant et la reconnaissance, qui n'ont pas de prise directe sur l'ordre matériel. L'intention de fonder une littérature régionale et la création du terme «Mauricie» constituent deux exemples frappants de cette stratégie visant une quête identitaire. Après avoir bâti le pays, il fallait le nommer et le fonder symboliquement par une littérature originale, actes créateurs situés dans le prolongement humanisé de la conquête sur la Nature. La création lexicale du régionyme Mauricie et le recours au discours historico-littéraire opèrent une double coupure dans le temps et dans l'espace, en nourrissant l'imaginaire collectif par un tri du passé et un balisage territorial. Toute collectivité engagée dans un processus d'identification fait appel à des mythes fondateurs, et il apparaît clairement que les Jean Nicolet, Pierre-Esprit Radisson et autres personnages glorifiés par la petite histoire trifluvienne ont joué ce rôle par la désignation de Joseph-G. Gélinas et d'Albert Tessier. De la même façon, les célébrations du Tricentenaire ont favorisé une rupture avec le quotidien au moyen d'une fusion collective réalisant l'esprit de la Fête où le passé mythique se substitue à une réalité oppressante par la magie d'un imaginaire réifié par les sens. La canonisation des héros trifluviens participe ainsi de la récupération du passé, de cet aménagement rétrospectif des données de l'Histoire que proposent les «pageants». Finalement, le projet littéraire de Tessier cherche lui aussi dans le passé des sécurités pour l'avenir.

Où peut-on situer le régionalisme mauricien par rapport aux grands courants d'idées contemporains au Canada français? Il faut préciser d'emblée que le discours régionaliste ne se réduit pas au ruralisme promu par certaines élites nationalistes ou cléricales qui 
voyaient le retour à la terre comme remède au chômage et conjuration contre les dangers moraux de la vie urbaine. Certes, Albert Tessier a célébré avec éloquence les vertus du mode de vie rural de même que l'environnement où il se déroule. Par ailleurs, il s'est efforcé de vanter l'industrie touristique et a chanté le Saint-Maurice, fournisseur d'énergie électrique, source du progrès selon lui. De son côté, LouisD. Durand n'a eu de cesse de promouvoir le commerce et l'industrie et a fait deux campagnes électorales sur la promesse d'un tunnel sous le fleuve pour l'union économique des deux rives. L'abbé Gélinas ne s'est jamais prononcé à ce sujet et Nérée Beauchemin, à l'instar de Frédéric Mistral, n'a posé qu'un regard nostalgique sur la vie ancestrale. Ces nuances paraissent nécessaires afin d'éviter l'enfermement du courant régionaliste dans le mythe du retour à la terre. Par ailleurs, le discours libéral de Louis-D. Durand, axé sur le développement économique et la promotion de la petite bourgeoisie, empêche le régionalisme mauricien d'être simplement étiqueté de discours clériconationaliste.

La biographie et les écrits de Tessier nous apprennent la fascination exercée sur lui par l'œuvre littéraire et la vision du monde de Frédéric Mistral. Tout comme le poète provençal, le prélat trifluvien s'est rigoureusement tenu à l'écart de toute revendication politique ou même économique. La Commission d'éducation nationale qu'il propose n'aurait constitué qu'un simple office de persuasion politique. De même, Durand, lui-même engagé dans l'action politique, n'a jamais revendiqué un quelconque pouvoir régional, comme la création d'entités administratives ou politiques spécifiques. Semblablement, la mise en branle de mécanismes qui auraient assuré un contrôle minimal des francophones sur l'économie régionale, comme la création de coopératives ou l'aide aux petites et moyennes entreprises, est une idée totalement étrangère au projet sociétal du régionalisme mauricien. Résignation devant une situation jugée définitive ou dédain des choses «matérielles»? Un peu des deux sans doute, mais peut-être que les émetteurs du régionalisme jugeaient que ce n'était pas à eux de le dire.

Une autre distinction doit être opérée. On serait tenté de confondre le régionalisme mauricien avec le régionalisme littéraire du Canada français mais, malgré des points communs, l'identification est encore là inexacte. Le régionalisme canadien-français faisait plutôt référence à une littérature nationale autochtone par opposition aux «exotistes» qui voulaient s'inspirer des courants contemporains de la littérature universelle. Certes, Tessier a énoncé un projet littéraire bien précis dans la foulée de ceux qui prônaient une littérature nationaliste tels Camille Roy, l'équipe de rédaction du Terroir et Lionel Groulx. Mais le régionalisme mauricien a dépassé le seul domaine de l'écrit: par le 
cinéma, la photographie, le théâtre (les «pageants du Tricentenaire»), la musique et l'action culturelle au sens large (la Société Le Flambeau), sans oublier l'école des sculpteurs, graveurs et artisans, ni le projet pédagogique que le régionalisme de Tessier inspirait.

C'est ailleurs qu'il faut situer exactement la doctrine régionaliste des années trente. Utilisant les canaux médiatiques les plus modernes pour l'époque, Albert Tessier et Louis-D. Durand ont transmis leur passion envers un paysage mauricien humanisé par l'action des missionnaires, des colonisateurs et des forestiers. Ainsi, ces deux idéologues bâtissaient le cadre spatio-temporel d'un imaginaire collectif en voie de constitution, dans un esprit de continuité et de tradition et non dans une volonté de changement structurel d'ordre politique ou économique. Sociologiquement, leur discours s'inscrit dans la stratégie de reproduction sociale d'une élite traditionnelle: former de futurs chefs de file en leur inculquant des schèmes de pensée et des systèmes de valeurs qui assureraient la pérennité de leur contrôle sur la société régionale.

Albert Tessier a prêché inlassablement une véritable vision pédagogique devant tous ses auditoires. C'est ce qu'avait bien compris Albert Pelletier pour qui la «doctrine» mauricienne n'était utile qu'aux écoliers... Pelletier avait vu juste, là où Jean-Charles Harvey ne percevait que le «terroirisme» et Olivar Asselin, un sujet de raillerie. On constate aisément qu'Albert Tessier a atteint son objectif pédagogique si l'on considère attentivement les buts de la Société Le Flambeau. Mais cette génération nouvelle, celle du Flambeau, aux prises avec une situation d'emploi difficile, développa la Société sans le secours de Tessier ni de l'évêché. Signe des temps: l'œuvre éducatrice du régionalisme mauricien fait naître une volonté d'émancipation vis-àvis ses propres objectifs. De son côté, la classe dirigeante anglophone respirait d'aise devant le caractère symbolique des gestes posés. Ainsi, sans s'en rendre compte, le régionalisme a servi les fins de la classe dominante rassurée de voir les Mauriciens s'affairer dans des débats intellectuels ou se promener en costumes du XVII ${ }^{e}$ siècle à la recherche d'un mythe fondateur, plutôt que reconquérir un espace économique occupé par le capital étranger. Il ne s'agit nullement d'en déduire qu'il ait existé effectivement une collusion entre les propriétaires des moyens de production (et leurs subalternes locaux) d'une part, et les régionalistes mauriciens d'autre part, mais la fatalité de ce résultat était inévitable compte tenu de la structure sociale de l'époque. Néanmoins, les considérations d'ordre socio-économique ne doivent pas faire oublier que le régionalisme mauricien a constitué, malgré sa fonction occultrice, un dynamisme capable de mobiliser dans un enthousiasme effectif les agents du développement régional. 
L'action régionaliste a constitué une force structurante en ce sens que les développeurs ont disposé d'un cadre de référence spatial pour l'énonciation de discours sociaux. De plus, cette saisie de l'espace régional dans sa réalité collective humaine, de même que dans sa durée, a permis de se libérer de l'angoisse engendrée par les mutations sociales rapides et la cassure opérée par la crise économique.

Ce courant d'idées a constitué le discours social dominant en Mauricie durant les années 1930-1940, discours certes énoncé par une petite bourgeoisie soucieuse d'assurer sa reproduction sociale, mais également discours fécond pour la dynamique régionale. Production avant tout symbolique, mais susceptible de dynamiser l'action collective au niveau régional. Le régionalisme mauricien a constitué un réservoir à fantasmes dans lequel puisa une génération confrontée à une réalité sociale difficile. Le besoin de renforcer un imaginaire en quête de symboles par des représentations s'appuyant sur la différence régionale s'est étayé d'une perception renouvelée de l'espace régional et des personnages historiques qui l'ont délimité à partir du XVII siècle.

Pas exactement courant littéraire ou revendication politique, tributaire mais distinct du clérico-nationalisme, influencé par le ruralisme, par le régionalisme littéraire et, dans une moindre mesure, par le libéralisme économique, la pensée du régionalisme mauricien n'est innovatrice que par sa présentation pédagogique appuyée sur les médias. Comme un éclair qui, durant quelques années à peine, a zébré le ciel de la Mauricie, l'élan d'enthousiasme régionaliste s'est évanoui avec le départ de Tessier pour la direction des écoles ménagères, le retrait de Durand de la sphère politique et le début du second conflit mondial. D'autres enjeux sociaux, à l'échelle planétaire cette fois-ci, ont radicalement transformé la dynamique des tensions sociales. Ce qui a constitué peut-être la singularité du mouvement, c'est la symbiose Histoire-Nature, présentée dans un cadre nationaliste et catholique, qui apportait une compensation apparemment illusoire mais victorieuse à la Crise. À défaut de contrôler l'instance économique, des hommes ont voulu défendre la souveraineté de leur imaginaire dans ce qui a constitué, en définitive, une compensation symbolique à l'échec économique. Par ailleurs, il serait intéressant, dans une recherche future, de retracer de possibles pratiques régionales dans les coopératives agricoles et le mouvement des Caisses Desjardins.

En définitive, le régionalisme en Mauricie a fonctionné comme une entreprise de restauration établie par une stratégie sociale et culturelle puisant dans l'imaginaire collectif les fondements sémantiques d'une utopie régionale. Cette restauration s'est développée à partir de 
l'appropriation symbolique d'un espace régional par une lecture mythifiante de l'Histoire et de la Nature, et par un acte de nomination régional, celui de la Mauricie. Tout à la fois discours occultant une aliénation économique, expression du clivage culturel entre les élites et la masse, idéologie de repli et élément structurant de la dynamique régionale, le régionalisme mauricien des années trente a reflété les tiraillements d'une collectivité régionale en gestation. 